\title{
BMJ Open Measuring the Outcomes of Maternal COVID-19-related Prenatal Exposure (MOM-COPE): study protocol for a multicentric longitudinal project
}

\author{
Livio Provenzi (D) , Serena Grumi, ${ }^{1}$ Roberto Giorda, ${ }^{2}$ Giacomo Biasucci, ${ }^{3}$ \\ Renza Bonini, ${ }^{3}$ Anna Cavallini, ${ }^{4}$ Lidia Decembrino, ${ }^{5}$ Bruno Drera, ${ }^{6}$ \\ Rossana Falcone, ${ }^{5}$ Elisa Fazzi, ${ }^{7,8}$ Barbara Gardella, ${ }^{9}$ Roberta Giacchero, ${ }^{10}$ \\ Renata Nacinovich, ${ }^{4,11}$ Camilla Pisoni, ${ }^{12}$ Federico Prefumo, ${ }^{7}$ Barbara Scelsa, ${ }^{13}$ \\ Maria Valentina Spartà, ${ }^{10}$ Pierangelo Veggiotti, ${ }^{13,14}$ Simona Orcesi, ${ }^{1,15}$ \\ Renato Borgatti, ${ }^{1,15}$ MOM-COPE Study Group
}

To cite: Provenzi L, Grumi S, Giorda $\mathrm{R}$, et al. Measuring the Outcomes of Maternal COVID-19-related Prenatal Exposure (MOM-COPE): study protocol for a multicentric Iongitudinal project. BMJ Open 2020;10:e044585. doi:10.1136/ bmjopen-2020-044585

- Prepublication history and supplemental material for this paper is available online. To view these files, please visit the journal online (http://dx.doi. org/10.1136/bmjopen-2020044585).

Received 08 September 2020 Revised 14 November 2020 Accepted 17 November 2020

Check for updates

(C) Author(s) (or their employer(s)) 2020. Re-use permitted under CC BY-NC. No commercial re-use. See rights and permissions. Published by BMJ.

For numbered affiliations see end of article.

Correspondence to

Dr Livio Provenzi;

livio.provenzi@mondino.it

\section{ABSTRACT}

Introduction COVID-19 is a highly infectious respiratory disease that rapidly emerged as an unprecedented epidemic in Europe, with a primary hotspot in Northern Italy during the first months of 2020. Its high infection rate and rapid spread contribute to set the risk for relevant psychological stress in citizens. In this context, motherinfant health is at risk not only because of potential direct exposure to the virus but also due to high levels of stress experienced by mothers from conception to delivery. Prenatal stress exposure associates with less-than-optimal child developmental outcomes, and specific epigenetic mechanisms (eg, DNA methylation) may play a critical role in mediating this programming association.

Methods and analysis We present the methodological protocol for a longitudinal, multicentric study on the behavioural and epigenetic effects of COVID-19-related prenatal stress in a cohort of mother-infant dyads in Northern Italy. The dyads will be enrolled at 10 facilities in Northern Italy. Saliva samples will be collected at birth to assess the methylation status of specific genes linked with stress regulation in mothers and newborns. Mothers will provide retrospective data on COVID-19-related stress during pregnancy. At 3, 6 and 12 months, mothers will provide data on child behavioural and socioemotional outcomes, their own psychological status (stress, depressive and anxious symptoms) and coping strategies. At 12 months, infants and mothers will be videotaped during semistructured interaction to assess maternal sensitivity and infant's relational functioning.

Ethics and dissemination This study was approved by the Ethics Committee (Pavia). Results will be published in peer-reviewed journals and presented at national and international scientific conferences.

Trial registration number NCT04540029; Pre-results.

\section{INTRODUCTION}

COVID-19 is a highly infectious respiratory disease that during the first months of 2020 quickly spread worldwide and reached the
Strengths and limitations of this study

- This study will provide both behavioural and epigenetic evidence of COVID-19 emergency psychological effects on mothers' and infants' health.

- This study allows to explore the role of target-gene methylation levels in mediating the association between maternal prenatal stress related to the COVID-19 emergency and infant developmental outcomes from birth to the 12-month follow-up.

- The planned enrolment of the control group may be hampered by the permanence of the COVID-19 emergency.

- The quasi-experimental method of the study does not allow to have a pre-pandemic measure of maternal mental health.

status of an unprecedented pandemic. ${ }^{1}$ The high risk of COVID-19 infection-together with the lack of clear scientific knowledge of this virus-represents a direct and indirect risk factor for citizens' psychological health. ${ }^{2}$ More specifically, the COVID-19related psychological stress should not be underestimated especially during specific sensitive developmental windows characterised by enhanced neuroplasticity and susceptibility to environmental exposures, such as pregnancy and neonatal life. ${ }^{4}$

In this scenario, the mother-infant health is at risk not only because of potential direct exposure to the virus ${ }^{5}$ but also due to high levels of stress continuously experienced by mothers from conception to delivery. ${ }^{6}$ The exposure to prenatal maternal stress predicts a wide variety of behavioural, emotional, cognitive and physical outcomes in the offspring. ${ }^{78}$ A recent literature review 
highlighted that stress experienced by mothers during pregnancy may negatively impact temperament, ${ }^{9}$ attentional processes ${ }^{10}$ and stress regulation ${ }^{11}$ during infancy and childhood. ${ }^{12}{ }^{13}$ Notably, not only the stress experienced during pregnancy, but also maternal regulatory processes, such as coping strategies, may play a crucial role in protecting children's developmental trajectories in the face of adverse environmental conditions. ${ }^{14}$

Epigenetic processes regulate the expression of genes and are especially sensitive to adverse environmental exposures. ${ }^{15} 16$ DNA methylation is a specific epigenetic mechanism that consists in the binding of a methyl group to dinucleotides of cytosine and guanine $(\mathrm{CpG}$ sites) and contributes to reduce gene expression (ie, gene silencing). ${ }^{17}$ Previous research documented that the early exposure of the fetus and the infant to sources of maternal stress may affect the methylation status of genes involved in stress regulation. This is especially true for prenatal stress exposure. Previous research has highlighted that maternal stress during pregnancy may associate with altered DNA methylation of genes involved in stress regulation, behaviour and emotional development. ${ }^{18}$ Target genome loci include CpG islands of the glucocorticoid receptor gene $(N R 3 C 1),{ }^{19}$ the serotonin transporter gene $(S L C 6 A 4),{ }^{20}$ the oxytocin receptor gene $(O X T R),{ }^{21}$ the brain-derived neurotrophic factor $(B D N F)^{22}$ and the inflammatory-related FKBP5 gene. ${ }^{23}$ Monk and colleagues ${ }^{23}$ reported that higher perceived maternal stress during pregnancy was associated with greater methylation of the NR3C1 gene in infants. Similar findings were reported by Cecil and collaborators ${ }^{24}$ for the OXTR gene. Altered methylation of the $S L C 6 A 4^{20}$ was also observed in mothers and infants after exposure to depressed mood in pregnancy. Notably, altered methylation of these genes may ultimately associate with less than optimal behavioural and neuroendocrine development in the offspring. ${ }^{25-29}$ This rapidly accumulating evidence suggests that the study of epigenetic mechanisms-and especially the status of stress-related genes-during pregnancy may provide critical insights on the potential role of prenatal stress on maternal and infant well-being.

Additionally, the literature to date also features examples of the epigenetic effects of large-scale disasters.
Behavioural epigenetic research conducted on the survivors of the World Trade Center terroristic attack in 2001 or healthcare emergencies such as the Canadian Ice Storm in 1998 highlighted the role played by stressrelated genes' epigenetic regulation in mediating the exposure to maternal stress on children outcomes later in life. ${ }^{30-32}$ As pregnancy is a sensitive period for developmental programming, investigating the epigenetic and behavioural consequences of COVID-19-related stress in infants of pregnant women who lived in the geographical area of Northern Italy—characterised by the most rapid virus spread in Europe-is a unique quasi-experimental condition that has relevant implications. Moreover, as previous studies were for the most part retrospective, ${ }^{33}$ the study of the epigenetic and behavioural consequences of COVID-19-related stress in pregnant women may provide innovative evidence from a prospective and longitudinal study design.

\section{METHODS AND ANALYSIS \\ Aims}

Main aims

Consistently, in the first months of 2020, we have launched a multicentre, longitudinal and prospective study, namely, the Measuring the Outcomes of Maternal COVID-19-related Prenatal Exposure (MOM-COPE) research project. The main goal of the MOM-COPE study is to assess the immediate effects of COVID-19-related stress exposure during pregnancy on the developmental outcomes of infants at birth and collect follow-up data throughout the first year of life, exploring the role of epigenetic regulation in mediating these effects.

\section{Specific aims and hypotheses}

The first specific aim is to investigate the association between prenatal maternal stress related to the COVID-19 epidemic and infants' behavioural and socioemotional outcomes from birth to 12 months of age (figure 1A). Previous studies have largely suggested that maternal prenatal stress may have long-lasting effects on infants' development during the first months of life. Consistently, we hypothesise that infants of mothers reporting higher
A.

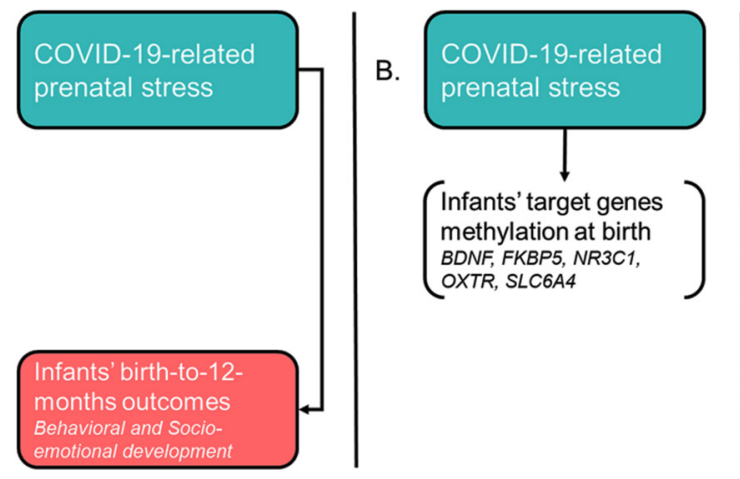

C.

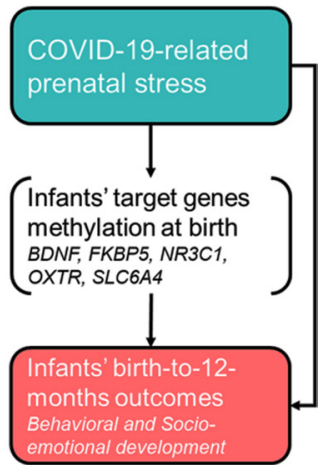

Figure 1 Overview of the specific aims of the MOM-COPE (Measuring the Outcomes of Maternal COVID-19-related Prenatal Exposure) study. 
levels of COVID-19-related stress during pregnancy will show evidence of less than optimal behavioural and socioemotional development.

The second specific aim is to investigate infant epigenetic signatures of COVID-19-related stress exposure by assessing the association between the levels of stress experienced by the mother during pregnancy and the DNA methylation level of target genes involved in stress regulation and socioemotional development: SLC6A4, ${ }^{34}$ $N R 3 C 1,{ }^{19} B D N F F^{35} O X T R^{22}$ and $F K B P 5^{36}$ (figure 1B). The regions of interest of the target genes have been previously shown to be susceptible to regulation (ie, hypermethylation profiles) in response to environmental exposures both in adults and infants. As such, we hypothesise that mothers who reported higher levels of COVID19-related prenatal stress and their infants would exhibit higher levels of methylation at the specific target genes assessed in the study.

Finally, the third specific aim is to explore the role of target-gene methylation level in mediating the association between maternal prenatal stress related to the COVID-19 emergency and infant developmental outcomes from birth to the 12-month follow-up (figure 1C). Previous research had limitations in testing prospective mediation models of target genes' methylation on the association between early adversities and later developmental outcomes in human infants and children. However, the longitudinal and prospective nature of this study supports the test of the hypothesis of a mediation role played by target genes' methylation levels on the link between maternal COVID-19-related prenatal stress and infants' behavioural and socioemotional outcomes.

\section{Additional exploratory aims}

As the exposure to stress during pregnancy may also affect maternal epigenetic regulation, ${ }^{37-39}$ we will also explore (1) the association between maternal COVID-19-related prenatal stress and the methylation status of specific target genes in mothers, (2) the concordance of infant and maternal methylation status of the same target genes and (3) the effect of prenatal COVID-19-related stress on maternal psychological health (ie, parenting stress, depression, anxiety) from delivery to the 12-month follow-up.

\section{Study design}

This multicentre, longitudinal and prospective study will enrol mother-infant dyads from 10 hospital facilities located in Northern Italy (figure 2). These facilities are geographically situated in the area that was first affected by the COVID-19 virus spread (eg, Pavia, Cremona, Lodi, Piacenza, Milano). The study includes two enrolment waves. First, the 'COVID-exposure pregnancy, CEP' wave will be enrolled from May to November 2020 in order to consecutively include mothers who were exposed to the COVID-19 emergency during the first 5-11 months of 2020. The second wave (ie, 'non-exposure pregnancy, NEP') will be recruited from May to November 2021 from the same hospital facilities in order to include mothers who have not experienced the same COVID-19 stress exposure.

\section{Enrolment}

Properly trained professionals will enrol mother-child dyads within 48 hours from birth at the participating neonatal units. Consecutive enrolment will be adopted, according to the inclusion and exclusion criteria reported in the Population section. Both mothers and fathers will provide written informed consent when possible. Nonetheless, due to the

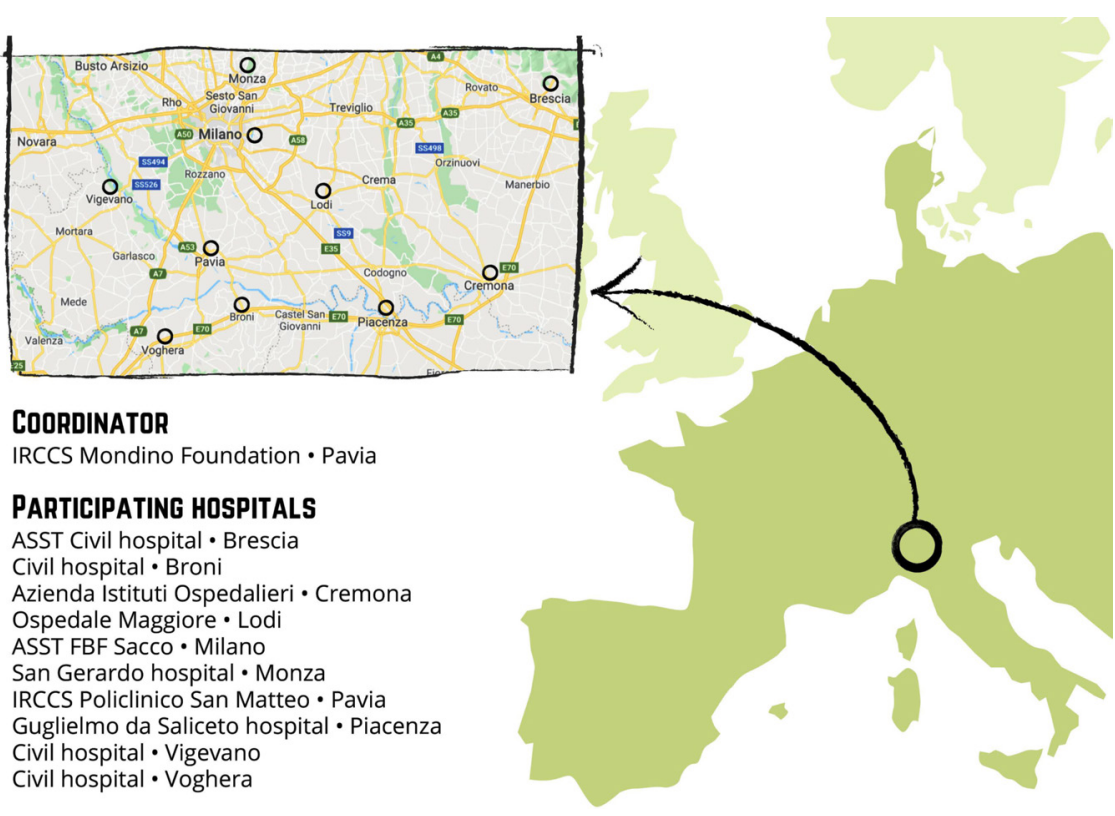

Figure 2 Distribution of the neonatal units participating to the MOM-COPE (Measuring the Outcomes of Maternal COVID-19related Prenatal Exposure) project in the geographical area of Northern Italy. Authors' own work. Original drawing by Marina Barello. 


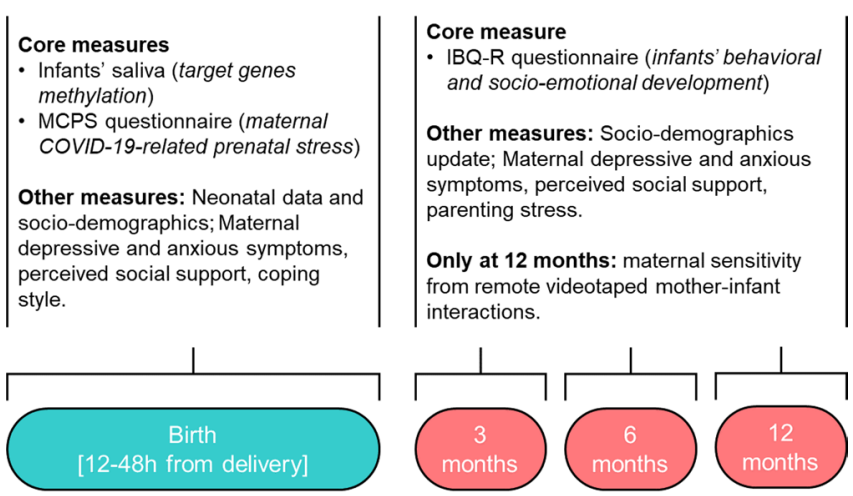

Figure 3 Schematic overview of the assessments included in the study design. Enrolment for wave 1 (COVID-exposure pregnancy, CEP) is planned from May to November 2020; enrolment for wave 2 (non-exposure pregnancy, NEP) is planned from May to November 2021. IBQ-R, Infant Behavior Questionnaire-Revised; MCPS, Maternal COVID-19-related Prenatal Stress.

restrictions adopted to contain and mitigate the COVID-19 virus spread, both parents will be allowed to provisionally provide online informed consent and they will provide proper signed informed consent when possible.

\section{Population}

Inclusion criteria are maternal Italian nationality or full mastery of Italian language in order to access the online questionnaires autonomously, no prenatal or perinatal risks, maternal age higher than 18 years and newborns' gestational age $\geq 37$ weeks. Mother-infant dyads will be not considered eligible to the study in the presence of the following exclusion criteria conditions: single-parent mothers, medically assisted conception, infants' genetic or metabolic comorbidities, perinatal injuries or sensory deficits.

\section{Procedures}

The methodology adopted in the MOM-COPE study aims to obtain reliable and valid measures to respond to the specific aims, while maximising the adherence to and respect of the Italian strategies for COVID-19 mitigation and containment. An overview of the study protocol and procedures is reported in figure 3. After obtaining informed consent, saliva samples will be taken from mothers and infants between 12 and 48 hours after delivery. This timing is meant to minimise the contamination of maternal fluids on the newborns' mouth and still maintain a consistent time window for biological assay among the different neonatal units involved. Neonatal data will be obtained from medical records.

At the same time, mothers will receive a web-link by email with the request to fill out the first set of questionnaires to collect sociodemographic information, maternal COVID19-related prenatal stress exposure, depressive and anxious symptoms, coping style and perceived social support. This set of questionnaires will be replicated at 3,6 and 12 months of age of the infant, with the addition of a brief parenting stress questionnaire and maternal report of infants' behavioural and socioemotional development. Finally, at 12 months of age, a videotaping of a semistructured mother-infant interaction will be obtained through video conferencing. The 8-minute mother-infant interaction will include normal faceto-face play with no toys $(2 \mathrm{~min})$, response to physical (train sound) and social (calling the infant name) auditory stimuli played remotely by the experimenter $(2 \mathrm{~min})$, a still-face episode (2min) and finally a normal face-to-face play with no toys $(2 \mathrm{~min})$. The use of the pacifier will not be allowed during this procedure. The mother will be instructed at the start of the video conference to prepare a setting in which she can sit in front of her infant placed in the infant seat, easily maintaining eye contact and close enough to facilitate hand contact.

\section{Core variables and measures for the specific aims}

\section{Maternal COVID-19-related prenatal stress exposure}

The Maternal COVID-19-related Prenatal Stress (MCPS) is a brief ad hoc self-report questionnaire that includes 28 items (a copy of the MCPS tool is included in online supplemental file S1). The items have been theoretically developed based on previous research on maternal stress response and coping in response to adverse experiences. ${ }^{40}{ }^{41}$ They capture four different dimensions of MCPS: physical exposure, emotional elaboration, cognitive elaboration and lifestyle. The dimensions were identified on the basis of the literature about stress response and coping strategies, which highlighted the relevance of the stressors' level of exposure together with the subject's response on the emotional, cognitive and behavioural levels. The items of each dimension were developed and selected on the basis of authors' clinical experience. Physical exposure $\left(\mathrm{MCPS}_{\mathrm{PE}}\right)$ includes seven dichotomous $(0$, no; 1 , yes) items capturing the maternal exposure to the risk or actual contagion for her and her close significant others. The MCPS $_{\mathrm{PE}}$ score will be obtained by summing the ratings of each item (raw score range: 0-7). Emotional elaboration $\left(\mathrm{MCPS}_{\mathrm{EE}}\right.$ ) includes six 5-point Likert scale items capturing the emotional and psychological stress response related to the COVID-19 emergency. The $\mathrm{MCPS}_{\mathrm{EE}}$ score will be obtained by computing a mean of the ratings obtained for each item (raw score range: 1-5). Cognitive elaboration $\left(\mathrm{MCPS}_{\mathrm{CE}}\right)$ includes nine 5-point Likert scale items related to coping strategies adopted to deal with COVID-19 stress and emotional burden. The MCPS $_{\mathrm{CE}}$ score will be obtained by computing a mean of the ratings obtained for each item (raw score range: 1-5). Lifestyle $\left(\mathrm{MCPS}_{\mathrm{LS}}\right)$ includes six 5-point Likert scale items targeting changes to habits and daily life behaviours to deal with the pandemic. The $\mathrm{MCPS}_{\mathrm{LS}}$ score will be obtained by computing a mean of the ratings obtained for each item (raw score range: $1-5$ ). The obtained raw scores will be standardised using z-score correction.

\section{Infant developmental outcomes}

Infants' behavioural and socioemotional development will be assessed through the 91-item maternal-reported Infant Behaviour Questionnaire-Revised (IBQ-R) short form ${ }^{42}$ which has proved satisfactory reliability and validity in the Italian context. ${ }^{44} 44$ The IBQ-R items are rated on a 7-point Likert scale and they are summarised into 14 scales, further 
summed up into three main factors: Surgency (scales: approach, activity level, high-intensity pleasure, perceptual sensitivity, smile and laughter, vocal reactivity), Negative Emotionality (scales: distress, fear, falling reactivity, sadness) and Orienting/Regulatory Capacity (scales: cuddliness, orienting, low-intensity pleasure, soothability).

\section{Target-gene methylation assessment}

The saliva will be collected from both mothers and infants using the OraCollect for Pediatrics kit OC-175 (DNA Genotek, Ottawa, Canada). Samples will be stored at room temperature by each neonatal unit and then shipped to the laboratory of the Scientific Institute IRCCS E. Medea (Bosisio Parini, Lecco, Italy) every 2 months. According to procedures adopted in previous research from the principal investigator, ${ }^{28} 293445$ genomic DNA will be extracted following manufacturer's protocols and its quality will be assessed using a Qubit fluorometer (Invitrogen, Thermo Fisher Scientific, Waltham, Massachusetts, USA). The methylation status of specific portions of the $B D N F, F K B P 5, N R 3 C 1$, OXTR and SLC6A4 genes will be assessed by PCR amplification of bisulfite-treated DNA followed by Next Generation
Sequencing (NGS) on a NEXTSeq-500 (Illumina, San Diego, California, USA). We will analyse one region of SLC6A4 (chr17:28562750-28562958, 20 CpGs); one region of NR3C1 (chr142763694-142764254, $44 \mathrm{CpGs);} \mathrm{one} \mathrm{region}$ of $B D N F$ (chr11:27723017-27723244, 14 CpGs); two regions of OXTR (promoter, chr3:8811489-8811838, 9 CpGs; intron 1, chr3:8810654-8810919, 13 CpGs), FKBP5 intron 5 (chr6: 35558405-35558550, 3 CpGs). A schematic summary of the CpG sites included for each gene is reported in figure 4.

\section{Other measures for the additional exploratory aims} Neonatal data

The following data will be collected from medical records: sex (0, female; 1 , male), gestational age (weeks), birth weight (grams), Apgar at minute 1 and 5 (range: 1-10), head circumference $(\mathrm{cm})$, length $(\mathrm{cm})$, type of delivery ( 0 , spontaneous vaginal; 1 , operative vaginal; 2 , elective caesarean; 3, emergency caesarean), small for gestational age (percentile), intrauterine growth restriction $(0$, no; 1 , yes), presence of morbidities ( 0 , no; 1 , yes), description of morbidities (text) and cord blood gas values (ie, $\mathrm{pH}$, $\mathrm{pO}_{2}, \mathrm{pcO}_{2}$, lactate, bicarbonate and base excess).

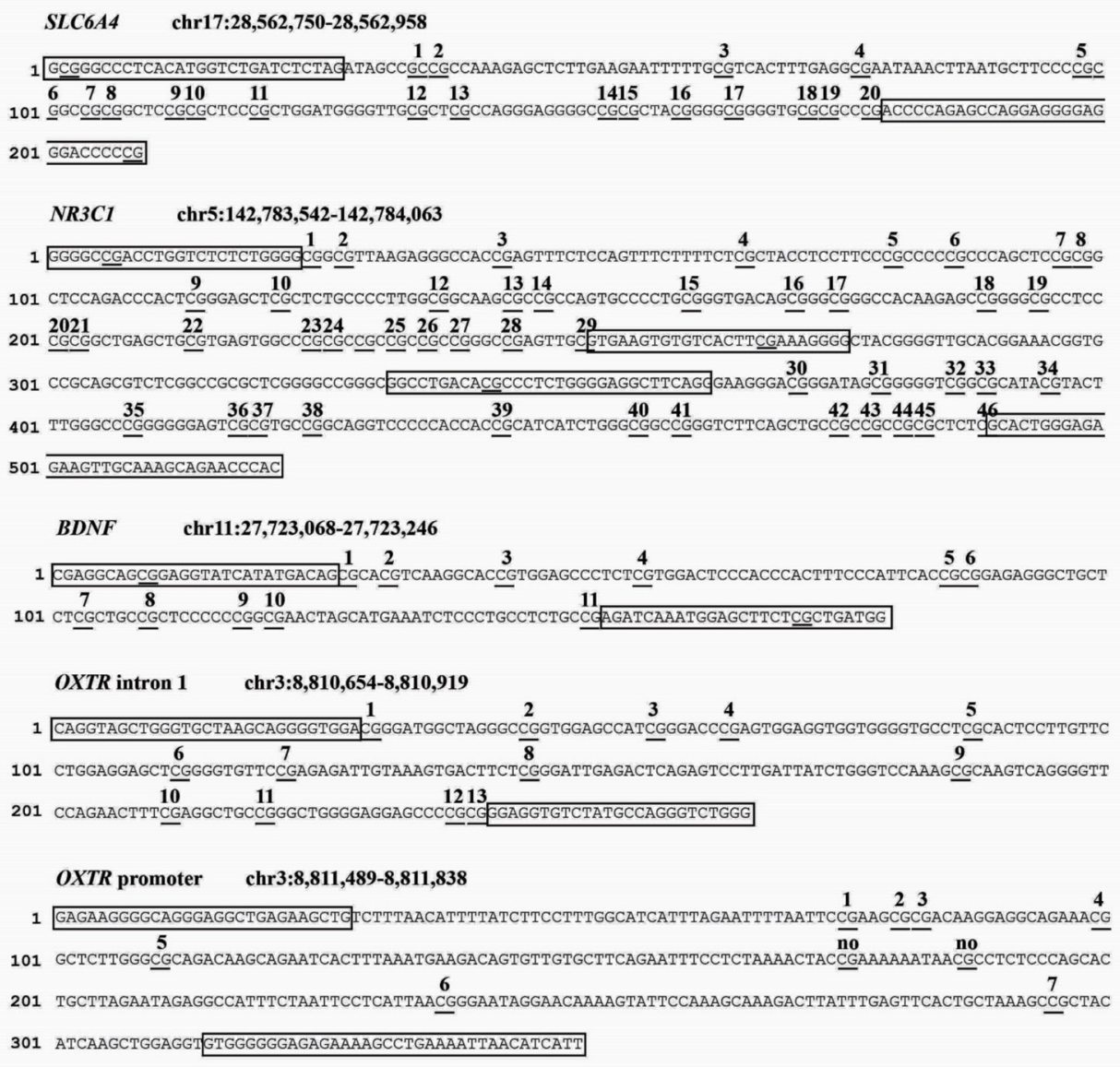

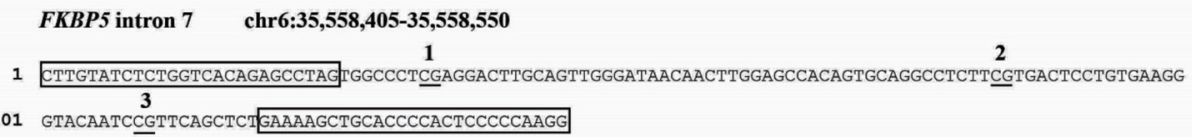

Figure 4 Schematic summary of the target genes' regions of interest for the present study. 


\section{Sociodemographic data}

Mothers will be asked to provide information about age (date of birth), educational level (highest educational degree) and occupational status.

\section{Maternal depressive symptoms}

The Beck Depression Inventory (BDI-II) ${ }^{46}$ is a 21 -item scale questionnaire widely used to assess depressive symptomatology. Each item is rated on a 4-point Likert scale. The BDI-II is commonly used in research on non-clinically depressed samples.

\section{Maternal anxious symptoms}

The 40-item State-Trait Anxiety Inventory (STAI-Y) ${ }^{47}$ includes a measure of transient anxiety symptoms (state anxiety: items 1-20) as well as a measure of anxiety traits (trait anxiety: items 21-40). Each STAI-Y item is rated on a 4-point intensity scale.

\section{Maternal parenting stress}

Parenting stress will be assessed through the Parenting Stress Index Short Form (PSI-SF).$^{48}$ It is a 36 -item selfreport questionnaire. It includes three subscales (Parental Distress, Parent-Child dysfunctional interaction and Difficult child) that are merged into a global parental stress score.

\section{Maternal perceived social support}

Social support will be measured through the Multidimensional Scale of Perceived Social Support (MSPSS ${ }^{49}$ which measures perceptions of support from three sources: family, friends and significant other. The scale consists of 12 items, with 4 items for each subscale.

\section{Maternal coping style}

Coping strategies will be measured through the Coping Orientation to Problems Experienced-New Italian Version (COPE-NVI). ${ }^{50}$ It is a 25-item self-report questionnaire that assesses preferential strategies used to cope with situations perceived as problematic. It includes five dimensions: Social support, Avoidance strategies, Positive attitude, Problem solving and Turning to religion.

\section{Maternal sensitivity}

Maternal sensitivity will be assessed from the videotaped mother-infant interactions obtained at 12-month infants' age using Global Rating Scales, II Edition (GRS) by Murray and colleagues (unpublished manual). This system has documented discriminant validity with populations of infants at low and high risk, as well as cross-cultural validation. ${ }^{28} 5152$ Maternal sensitivity is coded on a global rating scale ranging from 1 (the mother does not try to interpret the majority of infant's signals, she does not respect his/ her attempts to communicate and respond appropriately to his/her intentions; moreover, she may mock and laugh at him, with little or no sympathy at all) to 5 (the mother is highly sensitive for all the duration of the face-to-face interaction, producing frequent exaggeration, mirroring and affirming responses to the infant's signals).

\section{Statistical power and sample size estimation}

A minimum sample size of 105 subjects per group (alpha $=0.05$, beta $=0.05$, power $=0.95$, effect size, $d=0.50$ ) was estimated using $G^{*}$ Power software on the basis of previous research on the effect of maternal prenatal stress on infant development. ${ }^{53}$ Moreover, due to the longitudinal nature of the study, we estimate a $15 \%$ attrition rate at each of the three follow-up assessments, resulting in an initial oversampling of 160 mother-infant dyads per group (total sample size $=320$ ). Each neonatal unit will enrol at least 15-20 subjects for each group.

\section{Plan of statistical analyses}

$\mathrm{R}$ and IBM SPSS 26 packages will be used for the statistical analysis. Participants with more than $20 \%$ of missing data will be excluded from the analyses.

\section{Specific aims}

CEP and NEP groups will be preliminarily compared for sociodemographic and neonatal characteristics. In order to respond to the first specific aim of the MOM-COPE study, separated hierarchical regression models will be used to test the independent and joint predictive role of group and MCPS scales on infants' IBQ-R domain scores. The main additional sources of maternal stress have been removed through inclusion and exclusion criteria set out; however, potential confounders (eg, low socioeconomic conditions or traumatic events unrelated to the pandemic detected through self-report questionnaires) could not be completely excluded. Their intervening role will be preliminarily checked with Pearson's bivariate correlations and they will be included in the model if significantly associated with the outcome measures. In order to respond to the second specific aim, bivariate Pearson's correlations will be used to assess the association between MCPS scales and target genes' CpG methylation in the CEP and NEP groups. A cluster analysis will also be considered to explore the presence of multigene methylation patterns significantly linked with prenatal maternal stress related to the COVID-19 emergency. Finally, to respond to the third specific aim, general linear models will be used to assess the role of target genes' methylation at CpGs significantly linked with MCPS scales in mediating the association between maternal prenatal stress and infants' IBQ- R domains in both groups.

\section{Exploratory aims}

Pearson's bivariate correlations will be used separately in both groups (ie, CEP and NEP) to assess (1) the association between maternal COVID-19-related prenatal stress and the methylation status of specific target genes in mothers, (2) the concordance of infant and maternal methylation status of the same target genes and (3) the link between prenatal COVID-19-related stress and maternal psychological health (ie, parenting stress, depression, anxiety) at 3, 6 and 12 months' follow-up. 


\section{DISCUSSION}

\section{Limitations}

First, the quasi-experimental method of the study does not allow to have a pre-pandemic measure of maternal mental health. Second, the self-report nature of maternal stress measures may be partially biassed by the post hoc recall. Third, the planned enrolment of the NEP control group may be hampered by the enduring of COVID-19 emergency in the year 2021. Nonetheless, the recruitment of the NEP group will still occur after 1 year of emergency exposure and it will still be interesting to evaluate how maternal psychological response to the pandemic may vary between those exposed during the outbreak and those exposed after 12 months of being used to containment measures in daily life. Fourth, the inclusion criteria allow to control additional sources of maternal stress; however, they tend to define a medium-high socioeconomical portion of the eligible population.

\section{Expected results and implications}

This study can provide unprecedented relevant insights on the biochemical mechanisms underlying the impact of prenatal maternal stress on the infants, highlighting pathways of risk during a heightened period of neurodevelopmental plasticity and environmental sensitivity. First, as pregnancy is a specific sensitive window, ${ }^{4}$ mother and the newborn may be more susceptible to adverse environmental exposure as the COVID-19 emergency and this study will provide both behavioural and up-to-date epigenetic evidence of these effects. Second, this research project is warranted to contribute to the rapidly expanding and established field of behavioural epigenetics, providing novel prospective evidence about the intergenerational transmission of adverse environmental exposures. ${ }^{54}$ Third, by highlighting the presence of risk pathways for less-than-optimal behavioural and socioemotional development in infants of mothers exposed to the COVID-19-related stress during pregnancy, this research may also provide insights about the potential areas of preventive interventions in this population and in future similar events. Finally, this study will indirectly result in a large-scale repository of data about the early well-being and emotional development of infants in a region of Northern Italy that has been especially affected by the COVID-19 epidemic. As such, this study offers opportunity for longitudinal and multiple follow-up projects that may deepen the study of short-term and longterm impact of the COVID-19 epidemic on maternal and children health and well-being. Indeed, COVID-19 represents a case of social-economic-political-emotional stressor $^{55}$ that may determine biological consequences on newborns through both direct and indirect sequelae which affect the living conditions of mothers during pregnancy. For example, available evidence suggests that mothers are altering some aspect of their birth plan and they may be exposed to increased risk of depressed mood during the COVID-19 emergency. ${ }^{56}$

\section{Patient and public involvement}

Active engagement of families will be pursued through public dedicated website communications, digital content and leaflet reporting on the implications of the study and webinars. The involvement of relevant professional categories (eg, psychologists, neonatologists, paediatricians, obstetrics and gynaecologists) will be achieved by means of online webinars and meetings with the colleagues of the participating hospitals.

\section{ETHICS AND DISSEMINATION}

The study has received the approval of the Ethics Committee Pavia on 16 April 2020, Protocol number 20200037366. All the procedures are consistent with the Declaration of Helsinki ethical principles for research involving human subjects. ${ }^{57}$ The procedures do not imply any harm to the participating subjects nor any change to neonatal and mother-infant care programmes in place at the participating hospitals. The study protocol has also been registered on NIH Clinical Trials. The dissemination plan includes the presentation of findings at national and international scientific meetings, as well as the publication in scientific journals in the field of developmental psychology, neuroendocrinology, psychobiology, behavioural genetics and mother-infant health. The findings will also be disseminated to the public through reach-out activities involving families and healthcare specialists in order to promote a culture of mother-infant care that benefits from evidence-based research and further contribute to the long-term promotion of familycentred health and well-being.

\section{Author affiliations}

${ }^{1}$ Child Neurology and Psychiatry Unit, IRCCS Mondino Foundation, Pavia, Italy ${ }^{2}$ Biology Lab, Scientific Institute, IRCCS E. Medea, Bosisi Parini, Italy ${ }^{3}$ Pediatrics \& Neonatology Unit, Guglielmo da Saliceto Hospital, Piacenza, Italy ${ }^{4}$ Child and Adolescent Mental Health, San Gerardo Hospital, Monza, Italy

${ }^{5}$ Pediatric Unit and Neonatal Unit, Ospedale Civile di Vigevano, ASST di Pavia, Vigevano, Italy

${ }^{6}$ Neonatal Intensive Care Unit, Azienda Istituti Ospitalieri di Cremona, Cremona, Italy ${ }^{7}$ Department of Clinical and Experimental Sciences, University of Brescia, Brescia, Italy

${ }^{8}$ Unit of Child and Adolescence Neuropsychiatry, Azienda Ospedaliera Spedali Civili di Brescia, Brescia, Italy

${ }^{9}$ Department of Obstetrics and Gynecology, IRCCS Foundation Policlinico San Matteo, Pavia, Italy

${ }^{10}$ Department of Pediatrics, Lodi Hospital, Lodi, Italy

${ }^{11}$ School of Medicine and Surgery and Milan Center for Neuroscience, University of Milano Bicocca, Milano, Italy

${ }^{12}$ Neonatal Intensive Care Unit, IRCCS Foundation Policlinico San Matteo, Pavia, Italy

${ }^{13}$ Unit of Pediatric Neurology, Buzzi Children's Hospital, Milano, Italy

${ }^{14}$ Biomedical and Clinical Science Department, University of Milano, Milano, Italy

${ }^{15}$ Department of Brain and Behavioral Sciences, University of Pavia, Pavia, Italy

\section{Twitter Livio Provenzi @LivioProvenzi}

Acknowledgements The authors are thankful to the members of the MOM-COPE study group (listed under the heading "Collaborators"). Special thanks go also to Vanessa Manfredini, Giada Pettenati, and Elisa Rinaldi who provide support for data collection and at the time of this work were trainees at the IRCCS Mondino Foundation, Pavia, Italy. 
Collaborators MOM-COPE Study Group: Patrizia Accorsi, Rossana Bucci, Elisa Cavalleri, Laura Malerba, Paola Martelli, Mario Motta, Sonia Zatti, Emanuela Bertazzoli, Giovanna Centinaio, Maria Roberta Longo, Benedetta Chiara Pietra, Caterina Sabatini, Alberto Chiara, Giuliana Del Campo, Luisa Magnani, Dario Pantaleo, Arsenio Spinillo, Marco Zecca, Giulia Bensi, Elena Grossi, Cristiana Pavesi, Daniela Russo, Gaia Kullmann.

Contributors LP and RBor conceived the study protocol and the rationale and methods for the project; LP, SG and RBor conceived the paper and wrote a substantial part of it; RGio contributed to study design for the biological methods; GB, RBon, AC, LD, BD, RF, EF, BG, RGia, RN, CP, FP, BS, MVS, PV and SO contributed to define procedural decisions, study design and methodology, and definition of patients' inclusion criteria. All authors read and approved the final manuscript.

Funding The MOM-COPE study is funded by the Italian Ministry of Health through funds Cinque per Mille 2017 and Ricerca Corrente 2020.

Map disclaimer The depiction of boundaries on the map(s) in this article does not imply the expression of any opinion whatsoever on the part of BMJ (or any member of its group) concerning the legal status of any country, territory, jurisdiction or area or of its authorities. The map(s) are provided without any warranty of any kind, either express or implied.

Competing interests None declared.

Patient consent for publication Not required.

Provenance and peer review Not commissioned; externally peer reviewed.

Supplemental material This content has been supplied by the author(s). It has not been vetted by BMJ Publishing Group Limited (BMJ) and may not have been peer-reviewed. Any opinions or recommendations discussed are solely those of the author(s) and are not endorsed by BMJ. BMJ disclaims all liability and responsibility arising from any reliance placed on the content. Where the content includes any translated material, BMJ does not warrant the accuracy and reliability of the translations (including but not limited to local regulations, clinical guidelines, terminology, drug names and drug dosages), and is not responsible for any error and/or omissions arising from translation and adaptation or otherwise.

Open access This is an open access article distributed in accordance with the Creative Commons Attribution Non Commercial (CC BY-NC 4.0) license, which permits others to distribute, remix, adapt, build upon this work non-commercially, and license their derivative works on different terms, provided the original work is properly cited, appropriate credit is given, any changes made indicated, and the use is non-commercial. See: http://creativecommons.org/licenses/by-nc/4.0/.

ORCID iD

Livio Provenzi http://orcid.org/0000-0001-7424-8744

\section{REFERENCES}

1 Parodi SM, Liu VX. From containment to mitigation of COVID-19 in the US. JAMA 2020;323:1441-2.

2 Wang C, Horby PW, Hayden FG, et al. A novel coronavirus outbreak of global health concern. Lancet 2020;395:470-3.

3 Barello S, Palamenghi L, Graffigna G. Burnout and somatic symptoms among frontline healthcare professionals at the peak of the Italian COVID-19 pandemic. Psychiatry Res 2020;290:113129.

4 Linnér A, Almgren M. Epigenetic programming-The important first 1000 days. Acta Paediatr 2020;109:443-52.

5 Dong L, Tian J, He S, et al. Possible vertical transmission of SARS-CoV-2 from an infected mother to her newborn. JAMA 2020;323:1846-8.

6 Thompson LA, Rasmussen SA. What does the coronavirus disease 2019 (COVID-19) mean for families? JAMA Pediatr 2020;174:628.

7 Su Q, Zhang H, Zhang Y, et al. Maternal stress in gestation: birth outcomes and stress-related hormone response of the neonates. Pediatr Neonatol 2015;56:376-81.

8 Zhu P, Sun M-S, Hao J-H, et al. Does prenatal maternal stress impair cognitive development and alter temperament characteristics in toddlers with healthy birth outcomes? Dev Med Child Neurol 2014;56:283-9.

9 Laplante DP, Brunet A, King S. The effects of maternal stress and illness during pregnancy on infant temperament: project ice storm. Pediatr Res 2016;79:107-13.

10 Class QA, Abel KM, Khashan AS, et al. Offspring psychopathology following preconception, prenatal and postnatal maternal bereavement stress. Psychol Med 2014;44:71-84.
11 Field T, Diego M, Hernandez-Reif M, et al. Prenatal materna biochemistry predicts neonatal biochemistry. Int J Neurosci 2004;114:933-45.

12 Van den Bergh BRH, Mulder EJH, Mennes M, et al. Antenatal maternal anxiety and stress and the neurobehavioural development of the fetus and child: links and possible mechanisms. A review. Neurosci Biobehav Rev 2005;29:237-58.

13 Van den Bergh $\mathrm{BRH}$, van den Heuvel MI, Lahti M, et al. Prenatal developmental origins of behavior and mental health: the influence of maternal stress in pregnancy. Neurosci Biobehav Rev 2017:j. neubiorev.2017.07.003. doi:10.1016/j.neubiorev.2017.07.003

14 Cao-Lei L, Dancause KN, Elgbeili G, et al. Dna methylation mediates the effect of maternal cognitive appraisal of a disaster in pregnancy on the child's C-peptide secretion in adolescence: project ice storm. PLoS One 2018;13:e0192199.

15 Provenzi L, Giorda R, Beri S, et al. Slc6A4 methylation as an epigenetic marker of life adversity exposures in humans: a systematic review of literature. Neurosci Biobehav Rev 2016;71:7-20.

16 Weaver ICG, Cervoni N, Champagne FA, et al. Epigenetic programming by maternal behavior. Nat Neurosci 2004;7:847-54.

17 Hyman SE. How adversity gets under the skin. Nat Neurosci 2009;12:241-3.

18 Sosnowski DW, Booth C, York TP, et al. Maternal prenatal stress and infant DNA methylation: a systematic review. Dev Psychobiol 2018;60:127-39.

19 Palma-Gudiel H, Córdova-Palomera A, Leza JC, et al. Glucocorticoid receptor gene (NR3C1) methylation processes as mediators of early adversity in stress-related disorders causality: a critical review. Neurosci Biobehav Rev 2015;55:520-35.

20 Devlin AM, Brain U, Austin J, et al. Prenatal exposure to maternal depressed mood and the MTHFR C677T variant affect SLC6A4 methylation in infants at birth. PLoS One 2010;5:e12201.

21 Beery AK, McEwen LM, Maclsaac JL, et al. Natural variation in maternal care and cross-tissue patterns of oxytocin receptor gene methylation in rats. Horm Behav 2016;77:42-52.

22 Unternaehrer E, Meyer AH, Burkhardt SCA, et al. Childhood maternal care is associated with DNA methylation of the genes for brain-derived neurotrophic factor (BDNF) and oxytocin receptor (OXTR) in peripheral blood cells in adult men and women. Stress 2015;18:451-61.

23 Monk C, Feng T, Lee S, et al. Distress during pregnancy: epigenetic regulation of placenta glucocorticoid-related genes and fetal neurobehavior. Am J Psychiatry 2016;173:705-13.

24 Cecil CAM, Lysenko LJ, Jaffee SR, et al. Environmental risk, oxytocin receptor gene (OXTR) methylation and youth callous-unemotional traits: a 13-year longitudinal study. Mol Psychiatry 2014;19:1071-7.

25 Conradt E, Lester BM, Appleton AA, et al. The roles of DNA methylation of NR3C1 and 11 $\beta$-HSD2 and exposure to maternal mood disorder in utero on newborn neurobehavior. Epigenetics 2013;8:1321-9.

26 Stroud LR, Papandonatos GD, Salisbury AL, et al. Epigenetic regulation of placental NR3C1: mechanism underlying prenatal programming of infant neurobehavior by maternal smoking? Child Dev 2016;87:49-60.

27 Parent J, Parade SH, Laumann LE, et al. Dynamic stress-related epigenetic regulation of the glucocorticoid receptor gene promoter during early development: the role of child maltreatment. Dev Psychopathol 2017;29:1635-48.

28 Provenzi L, Fumagalli M, Giorda R, et al. Maternal sensitivity buffers the association between SLC6A4 methylation and socio-emotional stress response in 3-month-old full term, but not very preterm infants. Front Psychiatry 2017;8:171. doi:10.3389/fpsyt.2017.00171

29 Provenzi L, Fumagalli M, Scotto di Minico G, et al. Pain-Related increase in serotonin transporter gene methylation associates with emotional regulation in 4.5-year-old preterm-born children. Acta Paediatr 2020;109:1166-74.

30 Kuan P-F, Waszczuk MA, Kotov R, et al. An epigenome-wide DNA methylation study of PTSD and depression in world Trade center responders. Transl Psychiatry 2017;7:e1158.

31 Yehuda R, Engel SM, Brand SR, et al. Transgenerational effects of posttraumatic stress disorder in babies of mothers exposed to the world Trade center attacks during pregnancy. J Clin Endocrinol Metab 2005;90:4115-8.

32 Cao-Lei L, Massart R, Suderman MJ, et al. Dna methylation signatures triggered by prenatal maternal stress exposure to a natural disaster: project ice storm. PLoS One 2014;9:e107653.

33 Provenzi L, Borgatti R, Montirosso R. Why are prospective longitudinal studies needed in preterm behavioral epigenetic research? JAMA Pediatr 2017;171:92.

34 Fumagalli M, Provenzi L, De Carli P, et al. From early stress to 12-month development in very preterm infants: preliminary 
findings on epigenetic mechanisms and brain growth. PLoS One 2018;13:e0190602.

35 Kertes DA, Bhatt SS, Kamin HS, et al. BNDF methylation in mothers and newborns is associated with maternal exposure to war trauma. Clin Epigenetics 2017;9:68.

36 Yehuda R, Daskalakis NP, Bierer LM, et al. Holocaust exposure induced intergenerational effects on FKBP5 methylation. Biol Psychiatry 2016;80:372-80.

37 Oberlander TF, Weinberg J, Papsdorf M, et al. Prenatal exposure to maternal depression, neonatal methylation of human glucocorticoid receptor gene (NR3C1) and infant cortisol stress responses. Epigenetics 2008;3:97-106.

38 Kertes DA, Kamin HS, Hughes DA, et al. Prenatal maternal stress predicts methylation of genes regulating the hypothalamic-pituitaryadrenocortical system in mothers and newborns in the Democratic Republic of Congo. Child Dev 2016;87:61-72.

39 Provenzi L, Brambilla M, Scotto di Minico G, et al. Maternal caregiving and DNA methylation in human infants and children: systematic review. Genes Brain Behav 2020;19:1-11.

40 Cao-Lei L, Elgbeili G, Massart R, et al. Pregnant women's cognitive appraisal of a natural disaster affects DNA methylation in their children 13 years later: project ice storm. Transl Psychiatry 2015;5:e515.

41 Lobel M, Hamilton JG, Cannella DT. Psychosocial perspectives on pregnancy: prenatal maternal stress and coping. Soc Personal Psychol Compass 2008;2:1600-23.

42 Putnam SP, Helbig AL, Gartstein MA, et al. Development and assessment of short and very short forms of the infant behavior questionnaire-revised. J Pers Assess 2014;96:445-58.

43 Cozzi P, Putnam SP, Menesini E, et al. Studying cross-cultural differences in temperament in toddlerhood: United States of America (U.S.) and Italy. Infant Behav Dev 2013;36:480-3.

44 Montirosso R, Provenzi L, Fumagalli M, et al. Serotonin transporter gene (SLC6A4) methylation associates with neonatal intensive care unit stay and 3-Month-Old temperament in preterm infants. Child Dev 2016;87:38-48.

45 Provenzi L, Fumagalli M, Sirgiovanni I, et al. Pain-Related stress during the neonatal intensive care unit stay and SLC6A4 methylation in very preterm infants. Front Behav Neurosci 2015;9:1-9.
46 Beck AT, Steer RA, Carbin MG. Psychometric properties of the Beck depression inventory: twenty-five years of evaluation. Clin Psychol Rev 1988;8:77-100.

47 Spielberger CD, Gorsuch RL, Luschene R, et al. Manual for the State-Trait anxiety inventory. Palo Alto, CA: Consulting Psychologists Press, 1983.

48 Abidin RR. Parenting Stress Index: Manual, administration booklet, and research update. In: 'Research Update' presented at the Annual Meeting of the American Psychological Association 91st. 86. Charlottesville, VA, 1983.

49 Zimet GD, Powell SS, Farley GK, et al. Psychometric characteristics of the multidimensional scale of perceived social support. J Pers Assess 1990;55:610-7.

50 Carver CS, Scheier MF, Weintraub KJ. Assessing coping strategies: a theoretically based approach, 1989.

51 Gunning M, Halligan SL, Murray L. Contributions of maternal and infant factors to infant responding to the still face paradigm: a longitudinal study. Infant Behav Dev 2013;36:319-28.

52 Matricardi S, Agostino R, Fedeli C, et al. Mothers are not fathers: differences between parents in the reduction of stress levels after a parental intervention in a NICU. Acta Paediatr 2013;102:8-14.

53 Graignic-Philippe R, Dayan J, Chokron S, et al. Effects of prenatal stress on fetal and child development: a critical literature review. Neurosci Biobehav Rev 2014;43:137-62.

54 Provenzi L, Brambilla M, Borgatti R, et al. Methodological challenges in developmental human behavioral epigenetics: insights into study design. Front Behav Neurosci 2018;12:1-11.

55 Bogin B, Varea C. COVID-19, crisis, and emotional stress: a biocultural perspective of their impact on growth and development for the next generation. Am J Hum Biol 2020;32:e23474. doi:10.1002/ajhb.23474

56 Thayer ZM, Gildner TE. COVID-19-related financial stress associated with higher likelihood of depression among pregnant women living in the United States. Am J Hum Biol 2020:e23508. doi:10.1002/ ajhb.23508

57 World Medical Association. World Medical association Declaration of Helsinki. JAMA 2013;310:2191. 